\title{
Observation of the Low-Frequency Spectrum of the Water Dimer as a Sensitive Test of the Water Dimer Potential and Dipole Moment Surfaces
}

\author{
Raffael Schwan, Chen Qu, Devendra Mani, Nitish Pal, Lex van der Meer, Britta Redlich, \\ Claude Leforestier, Joel M. Bowman, * Gerhard Schwaab, and Martina Havenith*
}

\begin{abstract}
Using the helium nanodroplet isolation setup at the ultrabright free-electron laser source FELIX in Nijmegen (BoHeNDI@FELIX), the intermolecular modes of water dimer in the frequency region from 70 to $550 \mathrm{~cm}^{-1}$ were recorded. Observed bands were assigned to donor torsion, acceptor wag, acceptor twist, intermolecular stretch, donor torsion overtone, and in-plane and out-of-plane librational modes. This experimental data set provides a sensitive test for state-of-the-art water potentials and dipole moment surfaces. Theoretical calculations of the IR spectrum are presented using high-level quantum and approximate quasiclassical molecular dynamics approaches. These calculations use the full-dimensional ab initio WHHB potential and dipole moment surfaces. Based on the experimental data, a considerable increase of the acceptor switch and a bifurcation tunneling splitting in the librational mode is deduced, which is a consequence of the effective decrease in the tunneling barrier.
\end{abstract}

\section{Introduction}

Water is the most prominent solvent and of fundamental importance for chemistry and biology. After decades of

[*] Dr. R. Schwan, Dr. D. Mani, N. Pal, G. Schwaab, Prof. M. Havenith Physical Chemistry II, Department of Chemistry and Biochemistry Ruhr-Universität Bochum

Bochum (Germany)

E-mail: martina.havenith@rub.de

Dr. C. Qu, Prof. J. M. Bowman

Cherry L. Emerson Center for Scientific Computations and Department of Chemistry, Emory University

Atlanta, Georgia 30322 (USA)

E-mail: jmbowma@emory.edu

Dr. L. van der Meer, Dr. B. Redlich

Radboud University, Institute for Molecules and Materials

FELIX Laboratory

6525 ED Nijmegen (The Netherlands)

Prof. C. Leforestier

Institut Charles Gerhardt, UMR 5253 CNRS-UM-ENSCM

Université de Montpellier

Place Eugène Bataillon, 34090 Montpellier (France)

(2) Supporting information and the ORCID identification number(s) for iD the author(s) of this article can be found under: https://doi.org/10.1002/anie.201906048.

of (c) 2019 The Authors. Published by Wiley-VCH Verlag GmbH \& Co. $\mathrm{KGaA}$. This is an open access article under the terms of the Creative Commons Attribution Non-Commercial License, which permits use, distribution and reproduction in any medium, provided the original work is properly cited and is not used for commercial purposes. research, the goal of achieving a rigorous molecular description of the underlying intermolecular and intramolecular interactions is essentially at hand. This progress is built on the analysis of the convergence of the many-body representation of the total potential for water clusters, which shows that twobody interactions account for $80-90 \%$ and three-body interactions for almost all of the remaining interaction for electronic binding energies. ${ }^{[1-3]}$ Thus, the strategy for a general potential energy surface (PES) for arbitrary number of water monomers is based on rigorous two- and three-body interaction potentials, an accurate monomer potential, and for the final "polish" a treatment of four- and higher-body interactions, based on known polarizable models, for example, TTM3-F. ${ }^{[4]}$

The two-body interaction is completely described by the bonding and dynamics of the water dimer and so it is not surprising that theoretical analyses of the non-covalent bonding in the water dimer has a long history, dating from 1976 to the present. ${ }^{[5-9]}$ Much of what has been learned about the roles of permanent and induced multipole interactions, partial charge transfer, and Pauli repulsion in the water dimer is transferable to general non-covalent interactions between molecules. For this reason and also its central importance to understanding general water interactions the water dimer is arguably the most important molecular dimer in chemistry.

Beyond important qualitative understanding of the water dimer interaction, quantitative characterization of the water dimer potential has been a major challenge for theory. Early versions of such potentials were developed for rigid monomers and their forms were guided by known long-range and model repulsive interactions at short range. These potentials were optimized in conjunction with spectroscopic studies, which provided high-resolution spectra of intermolecular modes, in particular tunneling splittings in the spectrum that occur owing to equivalent minima. Several such potentials were reported in the early 2000s. ${ }^{[10-16]}$ However, these potentials do not describe the $\mathrm{O}-\mathrm{H}$ stretch modes of the water dimer, in particular the strongly red-shifted $\mathrm{H}$-bonded $\mathrm{O}-\mathrm{H}$ stretch. Also, they fall short in reproducing ab initio calculations of numerous low-lying stationary points of the water dimer that were reported in 2002. ${ }^{[17]}$ These later became important benchmarks for new generation of fully ab initiobased potentials for the water dimer.

A major step in this direction was made in 2007 with the introduction of the CC-pol water dimer potential. ${ }^{[18]}$ This is a non-linear least-squares fit to thousands of high-level $\operatorname{CCSD}(\mathrm{T}) / \mathrm{aug}$-cc-pVTZ energies for the water dimer with rigid monomers. This potential used numerous parameters 
with a physically based functional forms for the attractive and repulsive regions of the potential. (An SAPT potential for the three-body interaction, also for rigid monomers, completed this important pioneering work.) Quantum calculations of intermolecular energies and tunneling splittings, referred to as vibration-rotation tunneling (VRT) energies, using this $\mathrm{ab}$ initio potential were in excellent agreement with experiment. However, the CC-pol potential falls short in reaching the goal of a fully flexible ab initio potential for the water dimer. This was a challenge as such a potential is a function of 12 vibrational degrees of freedom and should be invariant with respect to permutation of $\mathrm{H}$ atoms. The first such potential was reported in $2006 .{ }^{[19]}$ It was a mathematical fit to roughly $20000 \mathrm{CCSD}$ (T)/aug-cc-pVTZ electronic energies (with the standard counterpoise correction), using permutationally invariant polynomials in Morse variables. Furthermore, a full-dimensional dipole moment surface, based on MP2/aug-cc-pVTZ dipole moments, at the same configurations as the electronic energies, was reported. A subsequent PES using roughly 30000 CCSD(T)/aug-cc-pVTZ (AVTZ) electronic energies was reported in 2008 and used in accurate quantum calculations of intermolecular energies and tunneling splittings in $\left(\mathrm{H}_{2} \mathrm{O}\right)_{2}$ and $\left(\mathrm{D}_{2} \mathrm{O}\right)_{2}{ }^{[20]}$ The final dimer PES in this series, referred to as HBB2, was reported in 2009. ${ }^{[21]}$ This PES reproduces the benchmark electronic dissociation energy of $4.98 \mathrm{kcal} \mathrm{mol}^{-1}$ and was used in a rigorous calculation of the observable dissociation energy, $D_{0}$, which was later confirmed experimentally. ${ }^{[22]}$ The HBB2 PES is the one used in the current calculations of the far-IR spectrum of the water dimer. Intensities are reported for the first time, using the dipole moment surface noted above. We note that the HBB2 potential is the two-body component of the more general potential, known as WHBB, ${ }^{[23]}$ which includes a full-dimensional 3-d potential. In this paper, we use WHBB and HBB2 interchangeably.

Subsequently, several other full-dimensional, ab initiobased water dimer potentials have been reported. One, reported in $2012^{[24]}$ and denoted CCpol-8s/f, is an extension of the last version of the rigid-monomer CC-pol potential, denoted CCpol-8s, ${ }^{[25]}$ plus a flexible SAPT potential. Another one ${ }^{[26]}$ follows the basic strategy used to develop the HBB2 potential. Namely, it used permutationally invariant polynomials. This potential is denoted MB-Pol. All of these dimer potentials give good agreement with experiment for VRT levels and tunneling splittings of $\left(\mathrm{H}_{2} \mathrm{O}\right)_{2}$ and the six fundamental intramolecular bend and vibrational energies. ${ }^{[24,26,27]}$ Very recently, quantum calculations of the second virial coefficient over the temperature range 300 to roughly $1200 \mathrm{~K}$ using HBB2, MB-Pol, and CCpol-8s/f were reported. ${ }^{[28]}$ In the low-temperature region the results from CCpol-8s/f and HBB2 are closer to each other and to experiment than are the results from MB-Pol. At higher temperature all potentials give similar and accurate results. Overall the results using CCpol-8s/f are the most accurate ones. In summary, these three flexible water dimer potentials appear to be highly accurate, at least for the properties examined to date. However, none of these potentials has been used in direct calculations of the IR spectrum in the far IR region, which is the focus herein.
Over several decades, high-resolution gas-phase measurements on the water dimer were carried out in the Saykally group, which enabled the development of accurate water dimer potentials. ${ }^{[9,29-32]}$ The water dimer is produced in a supersonic planar expansion and probed by several FIR laser (mixed with a tunable MW source) for frequencies $<150 \mathrm{~cm}^{-1}$ as well as lead salt diode lasers in the frequency range from 515 to $528 \mathrm{~cm}^{-1}$. This allowed to record spectra with a spectral resolution of $0.1 \mathrm{MHz}$, that is, $3 \times 10^{-6} \mathrm{~cm}^{-1}$ and to assign single vibrational rotational tunneling (VRT) transitions with resolved hyperfine splitting. Based on the spectra below $150 \mathrm{~cm}^{-1}$, it was possible to characterize the three feasible tunneling motions that connect the 8 degenerate minima on the 12-dim water potential (including 6 intramolecular and 3 intermolecular modes). However, despite of this enormous progress, for two degrees of freedom, which are very essential in characterizing bulk water dynamics, no information or only very limited information is available so far, owing to a lack of adequate laser sources. It is known now that the hydrogen bond breaking and reformation process in bulk water occurs via a pathway in which rotating water molecules break a hydrogen bond with an overcoordinated first shell neighbor to from an $\mathrm{H}$-bond with an undercoordinated neighbor. ${ }^{[33]}$ This water reorientation mechanism involves large-amplitude angular jumps. Thus, a precise measurement of the hindered rotation (denoted as libration) of, that is, the in-plane and out-of-plane tunneling mode in the water dimer is essential for a quantitative, precise prediction of the hydrogen bond network dynamics in the condensed phase. Whereas in high-resolution studies with each radiation source only small frequency ranges can be covered, for example, from 522 to $525 \mathrm{~cm}^{-1}$, in the present study the free electron laser FELIX allows coverage of the entire frequency range from $70 \mathrm{~cm}^{-1}$ to $3600 \mathrm{~cm}^{-1}$. In our study, the resolution of the free electron laser is restricted to the order of $1 \mathrm{~cm}^{-1}$. Thus, it is not possible to resolve single VRT transitions. However, we report a comprehensive study of all intermolecular modes of the water dimer in the frequency range from 100 to $600 \mathrm{~cm}^{-1}$ using helium nanodroplet isolation (HeNDI) spectroscopy. Based on our experimental results, we are able to deduce the acceptor switch (AS) tunneling for some of the excited states, including even the interchange tunneling. These full set of experimental data serves as a sensitive test for state-of-the-art water dimer potentials and dipole moment surfaces. For comparison we present the results of high-level quantum calculations fully coupled for the 6 intermolecular modes using a vibrational adiabatic potential for the 6 highfrequency intramolecular modes $(6 \mathrm{~d}+6 \mathrm{~d})$ and quasi classical molecular dynamics (QCMD) simulations using the HBB2 dimer potential and dipole moment surfaces. Thereby, it is possible to provide now an overview of all intermolecular modes including the higher frequency librational modes for water dimer as a sensitive test for one of the most recent and accurate water potentials. 


\section{Results and Discussion}

HeNDI spectroscopy is a powerful tool to record infrared spectra of single molecules and molecular clusters. ${ }^{[34-38]}$ The technique enables stepwise aggregation and the spectroscopic characterization of molecular aggregates with well-defined cluster sizes at ultracold temperatures of $0.37 \mathrm{~K} .{ }^{[39]}$ To study small water clusters in the FIR/THz region, we have combined a helium nandodroplet isolation spectrometer with the free-electron lasers (FELs), FEL-I and FEL-II, at the FELIX laboratory in Nijmegen. The experimental setup BoHeNDI@FELIX, combining the helium nanodroplet isolation technique with the ultrabright radiation source FELIX and covering the entire frequency region from 66 to $3600 \mathrm{~cm}^{-1}$, is described in detail in a previous publication. ${ }^{[40]}$

In HeNDI spectroscopy, the absorption is measured indirectly as the depletion of the ion current of a certain ionic fragment in the mass spectrum upon excitation of the embedded solute. In present study, we investigate water clusters, which are formed after sequential pick-up of single

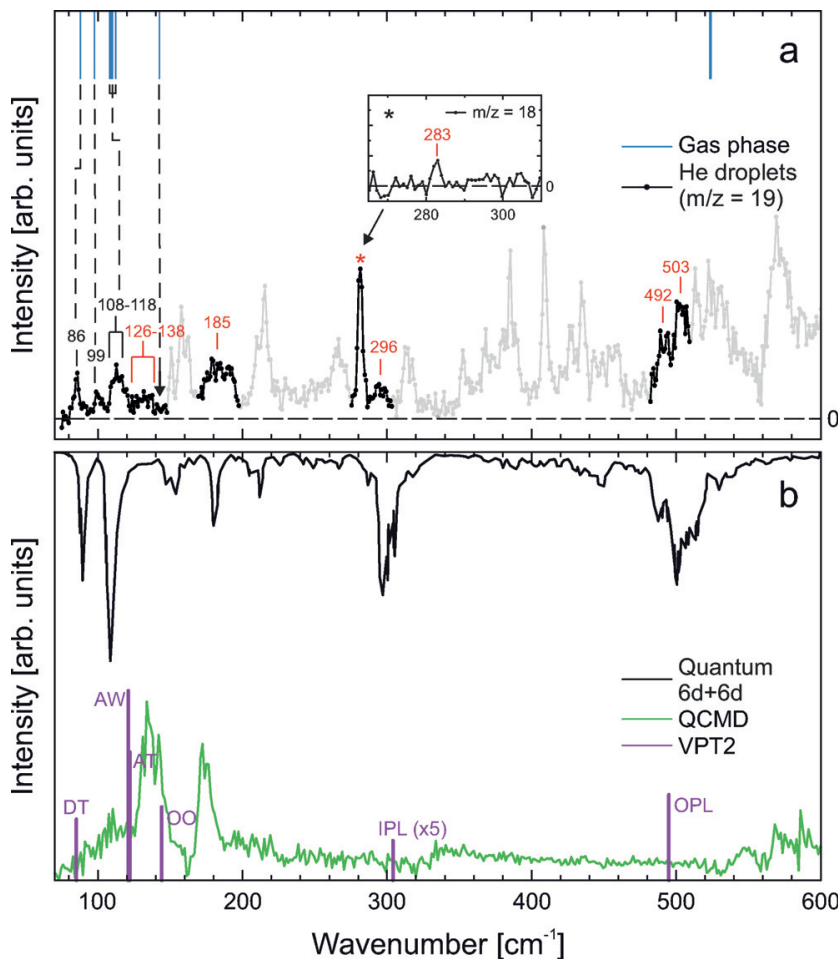

Figure 1. a) Overview of the FIR/THz depletion spectrum of the water cluster $\left(\mathrm{H}_{2} \mathrm{O}\right)_{2}$ in helium nanodroplets recorded at $m / z=19$ (lower trace, shown in gray). Bands that were assigned to water dimer are highlighted in black. The signal marked with a "**" is composed of water dimer, trimer, and higher clusters. For an assignment, we also recorded a depletion spectrum at $m / z=18$ (see inset), which minimizes water trimer contributions. Peak positions are given in $\mathrm{cm}^{-1}$. For comparison, we show the results of previous gas-phase studies $^{[29,31,43-45]}$ as blue lines (upper trace). Black numbers correspond to bands observed in previous gas-phase studies, while red numbers correspond to newly assigned bands. b) Predictions of the lowfrequency spectrum; black line: quantum $6 \mathrm{~d}+6 \mathrm{~d}$ (upper trace); green line: QCMD calculations (lower trace) both using the HBB2 12d ab initio potential and dipole moments; and purple sticks previous VPT2 calculations ${ }^{[46]}$ (lower trace). water molecules. IR absorption results in an evaporation of helium atoms and thereby in a decrease of ionization cross section which is detected at a loss of signal on the ionic fragment $\left[\mathrm{H}_{2} \mathrm{O}\right]^{+}$and $\left[\left(\mathrm{H}_{2} \mathrm{O}\right) \mathrm{H}\right]^{+}$at $\mathrm{m} / z=18$ and $\mathrm{m} / z=19$, respectively. An overview of the experimental low-frequency spectrum of water cluster in helium nanodroplets is shown in Figure 1 a (lower trace) in gray.

In previous studies, we could show that the depletion spectrum at $m / z=19$ is sensitive to the water dimer as well as trimer bands, while when recording at $m / z=18$, the intensity of water trimer signals is significantly reduced compared to those assigned to water dimer. ${ }^{[41]}$ Therefore, we recorded additional depletion spectra in the frequency range from 70 to $550 \mathrm{~cm}^{-1}$ at the mass of the ionic fragment at $\mathrm{m} / z=19$.at $\mathrm{m} /$ $z=18$. A definitive assignment of the cluster size is based on pressure-dependent intensity measurements, so-called pickup curves (see the Supporting Information). Bands which are assigned to water dimer bands are found in the following frequency regions: $70-145 \mathrm{~cm}^{-1}, 170-195 \mathrm{~cm}^{-1}, 265-310 \mathrm{~cm}^{-1}$, and $480-510 \mathrm{~cm}^{-1}$. These are highlighted in Figure 2 a, lower trace in black. We want to point out that the broad signal in the region from 170 to $195 \mathrm{~cm}^{-1}$ is attributed to a superposition of water dimer, water trimer as well as even larger water cluster bands. The signal at $283 \mathrm{~cm}^{-1}$ is found to be mainly due to water trimer. When scanning at $m / z=18$, we found a small peak which is assigned to the water dimer (see the Supporting Information).

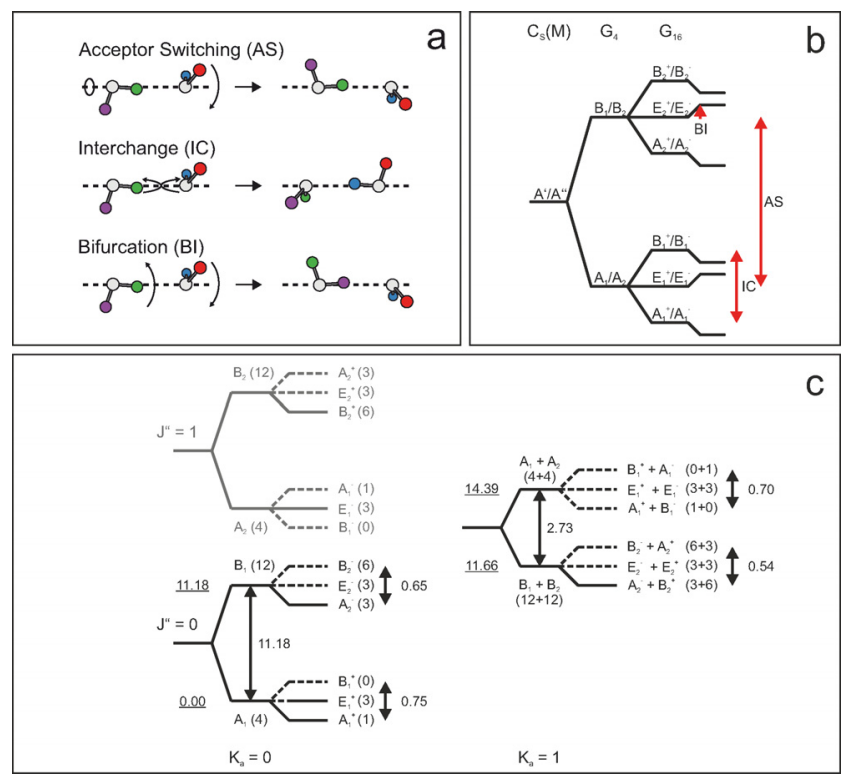

Figure 2. a) The three low-barrier tunneling motions of the water dimer. The acceptor switching (AS) involving an exchange of the two protons of the proton acceptor, the interchange (IC) exchanging the proton donor and proton acceptor, and the bifurcation (BI) tunneling. b) Owing to AS, each energy level is split into two energy levels. The symmetries are denoted in the irreducible representations of the $G_{4}$ MS group. IC tunneling leads to an additional splitting into three energy levels labeled within the irreducible representations of the $G_{16}$ MS group. BI causes a shift of the energy levels. c) VRT levels for $K_{\mathrm{a}}=0$ and $K_{\mathrm{a}}=1$ in the vibrational ground. At $0.37 \mathrm{~K}$, only VRT levels represented as solid lines are expected to be significantly populated. In parentheses, the spin statistical weights are shown. 
In Figure 1 a (upper trace) we display for comparison the intermolecular modes as observed before gas phase studies of the Saykally group. As mentioned before, these high-resolution studies are almost exclusively restricted to the frequency range below $150 \mathrm{~cm}^{-1}$, with the exception of one band at $524 \mathrm{~cm}^{-1} \cdot[29,31,42-45]$

In Figure $1 \mathrm{~b}$ upper trace, we show the results of our ab initio calculations using the $6 \mathrm{~d}+6 \mathrm{~d}$ quantum calculations (Figure 1b, upper trace) as well as predictions based upon quasi classical molecular dynamics (QCMD) simulations (Figure 1b, lower trace). Furthermore, in purple we display the results of previous vibrational perturbation theory of second order (VPT2) calculations. ${ }^{[4]}$ The details of the theoretical methods are described in the section Methods (see Supporting Information). The results are summarized in Table 1.

The water dimer exhibits three low-barrier tunneling motions. ${ }^{[47-49]}$ The three modes are displayed in Figure $2 \mathrm{a}$. These are denoted as acceptor switching (AS), interchange tunneling (IC), and bifurcation (BI) tunneling. The acceptor switching involves an exchange of the two protons in the hydrogen bonded acceptor monomer. The acceptor switching has the lowest barrier. If AS tunneling is feasible, the applicable molecular symmetry group is $G_{4}$, each rovibrational energy level is split into two energy levels (labeled as $A_{1} / B_{1}$ and $A_{2} / B_{2}$ ), see Figure $2 \mathrm{~b}$. Previously, the AS tunneling splittings in the vibrational ground state was deduced experimentally to be $11.18 \mathrm{~cm}^{-1}$ and $2.73 \mathrm{~cm}^{-1}$ for $K_{\mathrm{a}}^{\prime \prime}=0$ and $K_{\mathrm{a}}{ }^{\prime \prime}=1$ states, respectively. ${ }^{[13,42]}$

The interchange tunneling (IC) with a barrier of about $207 \mathrm{~cm}^{-1}$ exchanges the role of the hydrogen bond acceptor and the hydrogen bond donor water molecule. ${ }^{[30]}$ This tunneling causes an addition splitting of each state into three sublevels. These states can be labeled in the $G_{16}$ molecular symmetry group (see Figure $2 \mathrm{~b}$ ). In the vibrational ground state, the IC tunneling splitting is on the order of 0.54 to $0.70 \mathrm{~cm}^{-1}$. $^{[32]}$ The bifurcational (BI) tunneling describes a proton exchange of hydrogen bond donors. The tunneling mode involves an in plane librational motion of the donor and a flip of the acceptor monomer. In the ground state the barrier is on the order of $400 \mathrm{~cm}^{-1} \cdot{ }^{[30]}$ If $\mathrm{BI}$ tunneling is feasible, the energy levels of vibrational rotational tunneling states are shifted in energy.
Given the experimental frequency resolution of $1 \mathrm{~cm}^{-1}$ in our present study, in the ground state, only the AS tunneling splitting can be resolved. The two AS tunneling levels are also denoted as (1s) and (2s) state. Recent experimental ${ }^{[29,31,43-45]}$ and theoretical studies, ${ }^{[27,50]}$ showed that the IC tunneling splitting can be dramatically increased upon vibrational excitation. The selection rules in the $G_{16}$ molecular symmetry group are $\mathrm{A}_{1}{ }^{+} \leftrightarrow \mathrm{A}_{1}^{-}, \quad \mathrm{B}_{1}^{+} \leftrightarrow \mathrm{B}_{1}^{-}, \quad \mathrm{A}_{2}^{+} \leftrightarrow \mathrm{A}_{2}^{-}, \quad \mathrm{B}_{2}^{+} \leftrightarrow \mathrm{B}_{2}^{-}$, $\mathrm{E}^{+} \leftrightarrow \mathrm{E}^{-} \cdot{ }^{[47]}$ Note that in Figure $2 \mathrm{~b}$ the labeling of the E-states as either $E_{1}$ or $E_{2}$ is arbitrary and does not imply any further restrictions on selection rules. Transitions between $E$-states are allowed as long as the selection rule $+\leftrightarrow-$ is fulfilled.

In the present study, we expect a Boltzmann population of the VRT levels with a temperature of $0.37 \mathrm{~K}$. Thus, in general, only the lowest level of each symmetry will be populated. In Figure 2c the VRT levels for $K_{\mathrm{a}}=0$ and $K_{\mathrm{a}}=1$ in the vibrational ground state are shown. Vibration-rotationtunneling (VRT) levels, which are expected to be populated at $0.37 \mathrm{~K}$, are displayed as solid lines, while VRT states that are expected to be not significantly populated are represented as dashed lines (Figure 2c). Note that the $K_{\mathrm{a}}^{\prime \prime}=0(2 \mathrm{~s})$ and $K_{\mathrm{a}}^{\prime \prime}=1(2 \mathrm{~s})$ states are close in energy; thus both VRT levels will be populated.

In the present study, we focus on the low-frequency spectrum including all intermolecular modes. The intermolecular vibrational modes of the water dimer are either of $A^{\prime}$ or $A^{\prime \prime}$ symmetry. In principle, parallel $a$-type and perpendicular $c$-type transitions are allowed from the ground state to intermolecular mode with $A^{\prime}$ symmetry. Only perpendicular $b$-type transitions are allowed from the ground states to vibrationally excited modes with $A^{\prime \prime}$ symmetry. The relative intensity for parallel and perpendicular transitions can be predicted based on the ratio of the calculated parallel and perpendicular components of the transition dipole moments. These are listed in Table 1).

By comparison to previous gas-phase studies, the absorption bands in the frequency range from 70 to $145 \mathrm{~cm}^{-1}$ are assigned to the donor torsion, acceptor wag, and acceptor twist (see Table 1). An energy diagram for each of these modes along with the experimentally observed transitions in the gas phase as well as the transitions observed in the present paper are displayed in the Supporting Information.

The band at $86 \mathrm{~cm}^{-1}$ is assigned to the perpendicular $K_{\mathrm{a}}=$ $1 \leftarrow 0$ (1s) transition from the ground state to the excited donor torsion ( $A^{\prime \prime}$ symmetry), in excellent agreement with previous gas-phase measurements, which yielded a band center at $87.7 \mathrm{~cm}^{-1} \cdot{ }^{[43]}$ Thus we conclude, that the matrix shift caused by the helium nanodroplet environment is very small, that is, on the order of $1 \mathrm{~cm}^{-1}$ or less. In gas-phase studies, the IC tunneling splitting of the donor torsion $K_{\mathrm{a}}{ }^{\prime}=$ $1(1 \mathrm{~s})$ states has been determined to be $1.11 \mathrm{~cm}^{-1} \cdot{ }^{[43]}$ Taking into account the resolution of our experiment of $1 \mathrm{~cm}^{-1}$, for this mode, the IC tun- 
neling is expected to cause only a broadening of the observed band, no splitting can be resolved here. This $K_{\mathrm{a}}=1 \leftarrow 0(1 \mathrm{~s})$ transition marks the lower limit of the experimentally accessible frequency range of FELIX. Therefore, the $K_{\mathrm{a}}=$ $1 \leftarrow 0(2 \mathrm{~s})$ transition, which is predicted to be even about $7 \mathrm{~cm}^{-1}$ lower in frequency, ${ }^{[27,50]}$ as well as the $K_{\mathrm{a}}=0 \leftarrow 1(2 \mathrm{~s})$ transition cannot be observed here.

For transitions from the ground state to the acceptor wag ( $A^{\prime}$ symmetry), both, parallel as well as perpendicular transitions are allowed. We assign the signal at $99 \mathrm{~cm}^{-1}$ to a superposition of parallel $K_{\mathrm{a}}=0 \leftarrow 0(2 \mathrm{~s})$ as well as perpendicular $K_{\mathrm{a}}=0 \leftarrow 1(2 \mathrm{~s})$ transitions to the excited acceptor wag, in agreement with previous results in the gas phase (see the Supporting Information) ${ }^{[43]}$ More specifically, the broad band at $108-118 \mathrm{~cm}^{-1}$ can be attributed to a superposition of different bands, the $K_{\mathrm{a}}=0 \leftarrow 0(1 \mathrm{~s}), K_{\mathrm{a}}=1 \leftarrow 0(1 \mathrm{~s}), K_{\mathrm{a}}=1 \leftarrow 0$ (2s), and $K_{\mathrm{a}}=1 \leftarrow 1(2 \mathrm{~s})$ (see the Supporting Information). In previous studies, ${ }^{[43]}$ it was found that the IC tunneling splitting is increased to 2.95 and $5.24 \mathrm{~cm}^{-1}$, respectively, in the excited vibrational state. Thus, we expect two subbands, that is, the $K_{\mathrm{a}}=0 \leftarrow 0(1 \mathrm{~s})$ and $K_{\mathrm{a}}=1 \leftarrow 0(1 \mathrm{~s})$ transition from the ground state to the acceptor. However, owing to the plethora of bands in this frequency region, this splitting cannot be further resolved here.

The acceptor twist mode has $A^{\prime \prime}$ symmetry, thus only perpendicular transitions from the ground state to the excited acceptor twist are expected. Based on the results of previous gas-phase studies, ${ }^{[43]}$ the broad signal at $108-118 \mathrm{~cm}^{-1}$ is assigned to a $K_{\mathrm{a}}=0 \leftarrow 1(2 \mathrm{~s})$ transition (see also the Supporting Information). Based upon theoretical predictions, ${ }^{[27,50]}$ the $K_{\mathrm{a}}=1 \leftarrow 0(1 \mathrm{~s})$ and $K_{\mathrm{a}}=1 \leftarrow 0(2 \mathrm{~s})$ bands are expected at approximately 125 and $142 \mathrm{~cm}^{-1}$, respectively. In our study we observe a broad band between $126-138 \mathrm{~cm}^{-1}$. We assign this to an overlap of two transitions, that is, the $K_{\mathrm{a}}=1 \leftarrow 0(1 \mathrm{~s})$ and $K_{\mathrm{a}}=1 \leftarrow 0(2 \mathrm{~s})$ transitions from the ground state to the excited acceptor twist. Thus, we conclude that the AS tunneling splitting must be overestimated by theoretical predictions. Based on our experimental results, we propose that both vibrational rotational tunneling bands lie closer in energy, that is, they are separated by less than $9 \mathrm{~cm}^{-1}$.

The transitions from the ground state to the intermolecular stretch are predicted to lie in the region from approximately 140 to $150 \mathrm{~cm}^{-1} \cdot{ }^{[27,50]}$ In the gas phase,${ }^{[31,44]}$ only the $K_{\mathrm{a}}=0 \leftarrow 0(2 \mathrm{~s})$ and $K_{\mathrm{a}}=0 \leftarrow 1(2 \mathrm{~s})$ bands at about $142 \mathrm{~cm}^{-1}$, could be were observed, see the Supporting Information. Furthermore, bands at about $142 \mathrm{~cm}^{-1}$ have been reported, which were tentatively assigned to transitions from the ground state to the donor torsion overtone ( $A^{\prime}$ symmetry).${ }^{[1,44]}$ In our experimental spectrum we find a very weak signal, indicated by an arrow in Figure 1 a), which we assign to a transition to the intermolecular stretch. Unfortunately, the region around $146 \mathrm{~cm}^{-1}$ is dominated by signals attributed to water trimer. Using the ab initio $6 d+6 d$ potential (see Figure $1 \mathrm{~b}$, upper trace), we predict a small intensity for transitions to the intermolecular stretch of the water dimer compared to these water trimer bands. This explains why the frequency range between 140 and $150 \mathrm{~cm}^{-1}$ is dominated by trimer lines.
As was stated before, while in previous gas-phase studies transitions at $142 \mathrm{~cm}^{-1}$ were tentatively assigned to the donor torsion overtone mode, an unambiguous assignment is difficult owing to the fact that the intermolecular stretch is close in energy. In our present study, using HeNDI spectroscopy, we observe for the first time a broad signal at 170$195 \mathrm{~cm}^{-1}$, which is assigned to a transitions from the ground state to the upper (2s) tunneling of the donor torsion overtone mode. The pickup curve at $185 \mathrm{~cm}^{-1}$ confirms significant contributions of the water dimer (see the Supporting Information). By comparison to the spectrum based on the ab initio $6 d+6 d$ potential (see Figure $1 b$, upper trace), this signal can be unambiguously assigned to the donor torsion overtone. Upon excitation in the donor torsion overtone mode, the AS tunneling, that is, the energy difference between the $1 \mathrm{~s}$ and $2 \mathrm{~s}$ level is expected to be considerably increased. The $K_{\mathrm{a}}{ }^{\prime}=0(2 \mathrm{~s})$ state is predicted at an energy of $185 \mathrm{~cm}^{-1}$ with an increased IC tunneling splitting of $18.2 \mathrm{~cm}^{-1} \cdot{ }^{[50]}$ Here, we assign the experimentally observed

\section{Donor Torsion Overtone}

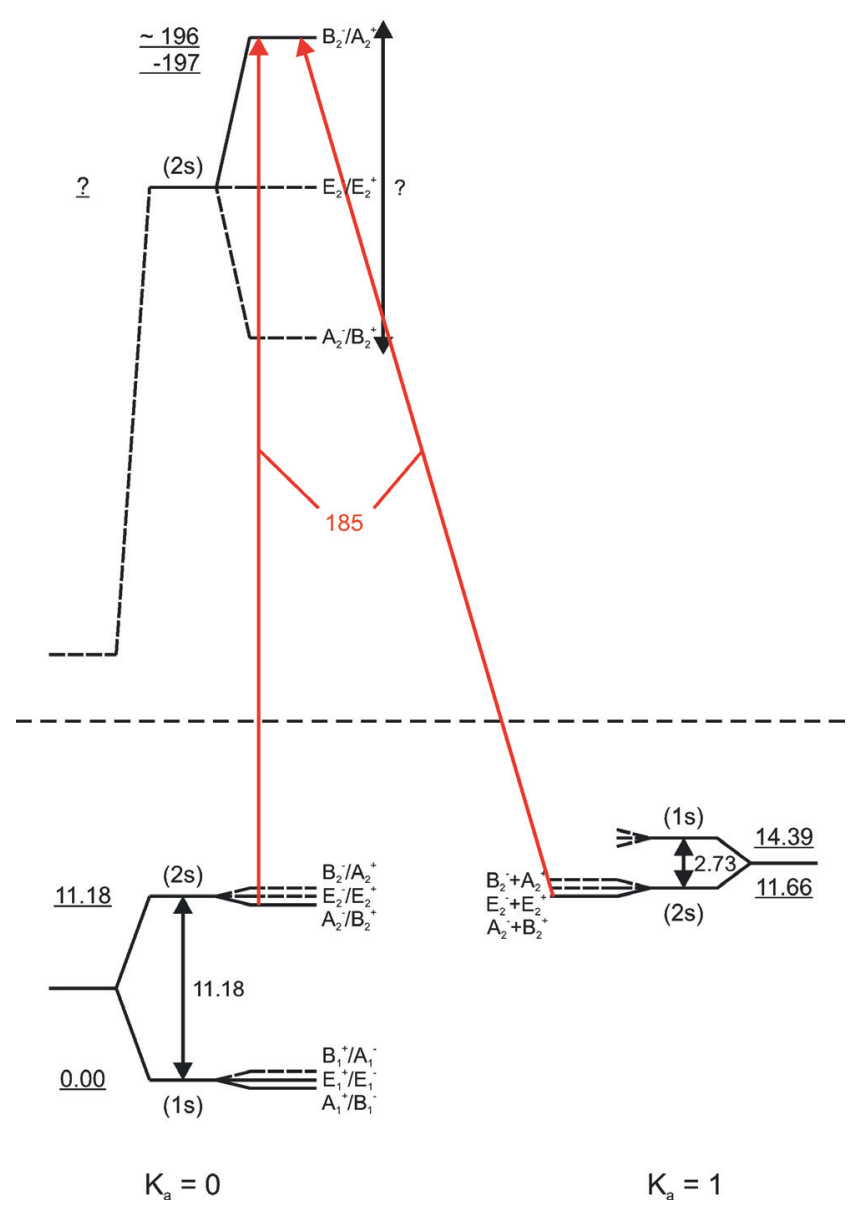

Figure 3. Donor torsion overtone (DT) ${ }^{2}$ VRT energy level diagram. Parallel and perpendicular transitions are shown as vertical and diagonal arrows, respectively; red arrows: present study only. For bands observed in the present study, the transition frequencies are given (in red). All frequencies and energies are given in $\mathrm{cm}^{-1}$. 
band to a transitions to the $K_{\mathrm{a}}{ }^{\prime}=0(2 \mathrm{~s})$ state of the donor torsion overtone. For both the $K_{\mathrm{a}}=0 \leftarrow 0(2 \mathrm{~s})$ and $K_{\mathrm{a}}=0 \leftarrow 1$ (2s) transitions, we expect transitions to the $A_{2}^{-} / B_{2}^{+}$subbands in this frequency range (see Figure 3 ). Based on our experiment, we deduce an energy of the $K_{\mathrm{a}}{ }^{\prime}=0\left(2 \mathrm{~s}, \mathrm{~A}_{2}{ }^{-} / \mathrm{B}_{2}{ }^{+}\right)$to be $196-197 \mathrm{~cm}^{-1}$, which is $10 \mathrm{~cm}^{-1}$ higher than the predictions. This implies that the AS tunneling splitting is even more increased than previously predicted.

So far, the frequency range between $150 \mathrm{~cm}^{-1}$ and $520 \mathrm{~cm}^{-1}$ could not be accessed experimentally. In this frequency range, the in-plane and out-of-plane librational modes are predicted, which provide a most sensitive test of the accuracy of state-of-the-art water dimer potential surfaces, especially in respect to the large amplitude angular motion. Using HENDI spetroscopy and cluster size determination via pick-up curves, we assign two bands centered at 283 and $296 \mathrm{~cm}^{-1}$ to the in-plane librational mode ( $A^{\prime}$ symmetry) of water dimer. In principle, both parallel and perpendicular transitions are allowed for the in-plane libration (Figure 4). However, the parallel component of the transition dipole moment is predicted to be much weaker than the perpendicular component (see Table 1). Therefore, the signal at $283 \mathrm{~cm}^{-1}$ is assigned to the $K_{\mathrm{a}}=0 \leftarrow 1(2 \mathrm{~s})$ and the signal at $296 \mathrm{~cm}^{-1}$ to the $K_{\mathrm{a}}=1 \leftarrow 0(1 \mathrm{~s})$ band. Depending on the magnitude of the AS tunneling splitting in the excited

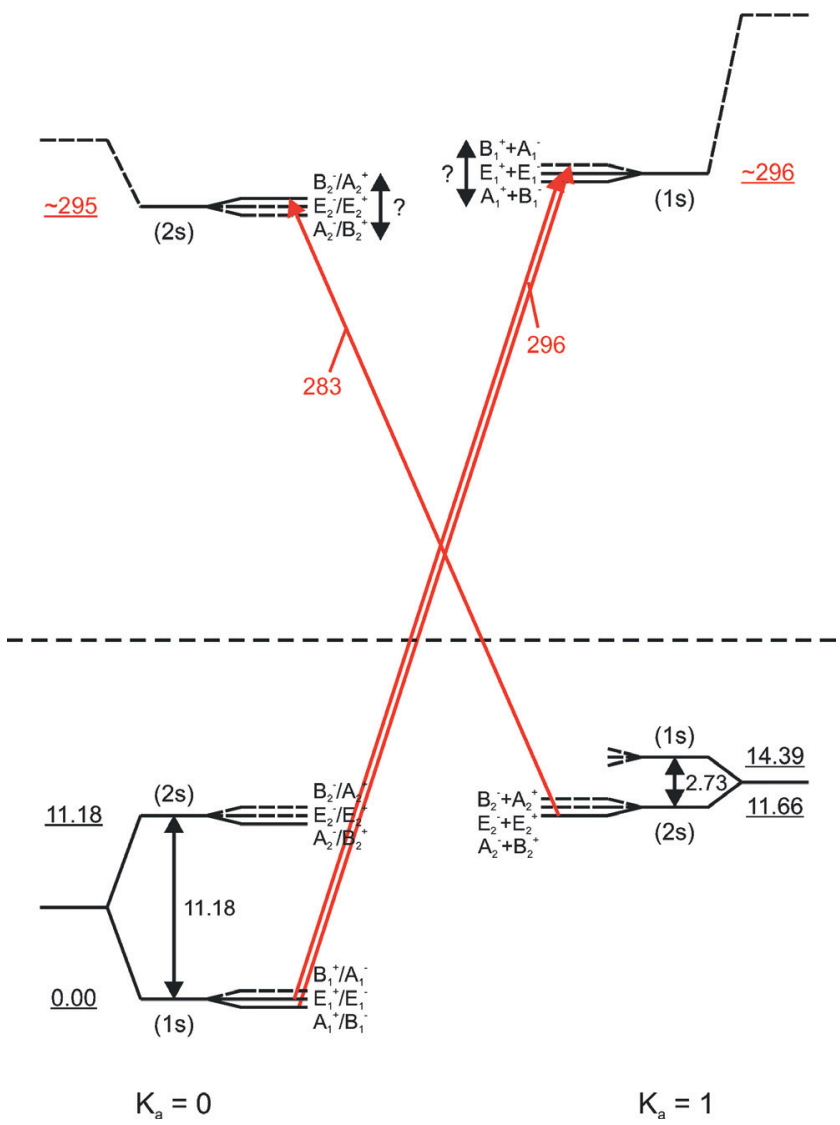

Figure 4. In-plane libration (IPL) VRT energy-level diagram. Parallel and perpendicular transitions are shown as vertical and diagonal arrows, respectively; red arrows: present study only. For bands observed in the present study, the transition frequencies are given (in red). All frequencies and energies are given in $\mathrm{cm}^{-1}$. vibrational states, the $K_{\mathrm{a}}=1 \leftarrow 0(2 \mathrm{~s})$ band might overlap with either the $K_{\mathrm{a}}=0 \leftarrow 1(2 \mathrm{~s})$ or the $K_{\mathrm{a}}=1 \leftarrow 0(1 \mathrm{~s})$ band.

In the frequency range of the out-of-plane librational mode ( $A^{\prime \prime}$ symmetry), we detect two signals at 492 and $503 \mathrm{~cm}^{-1}$ (see Table 1 ). In principle, only perpendicular transitions are allowed for the out-of-plane libration. However, in a recent study parallel as well as perpendicular transitions could be observed. ${ }^{[45]}$ For $E$-states both transitions are allowed as long as the selection rule $+\leftrightarrow-$ holds. We assign the band at $492 \mathrm{~cm}^{-1}$ to the $K_{\mathrm{a}}=0 \leftarrow 1(2 \mathrm{~s})$ transition and the band at $503 \mathrm{~cm}^{-1}$ to the $K_{\mathrm{a}}=0\left(E_{2}\right) \leftarrow 0\left(E_{1}\right)$ transition from the ground state to the excited out-of-plane librational mode (see Figure 5). Based on our measurements we can deduce that the splitting between the $K_{\mathrm{a}}{ }^{\prime}=0\left(2 \mathrm{~s}, \mathrm{~A}_{2}{ }^{+} / \mathrm{B}_{2}{ }^{-}\right)$and $K_{\mathrm{a}}{ }^{\prime}=0\left(2 \mathrm{~s}, \mathrm{E}_{2}{ }^{+} / \mathrm{E}_{2}{ }^{-}\right)$is small $\left(1 \mathrm{~cm}^{-1}\right)$. Furthermore, we are able to prove now information on the energy of the $K_{\mathrm{a}}{ }^{\prime}=0(2 \mathrm{~s})$ state, which lies at $503 \mathrm{~cm}^{-1}$. Based upon the results of our study and the previous gas-phase study, we can deduce the size of the acceptor switch (AS) tunneling splitting in the outof-plane librational mode which amounts to the energy

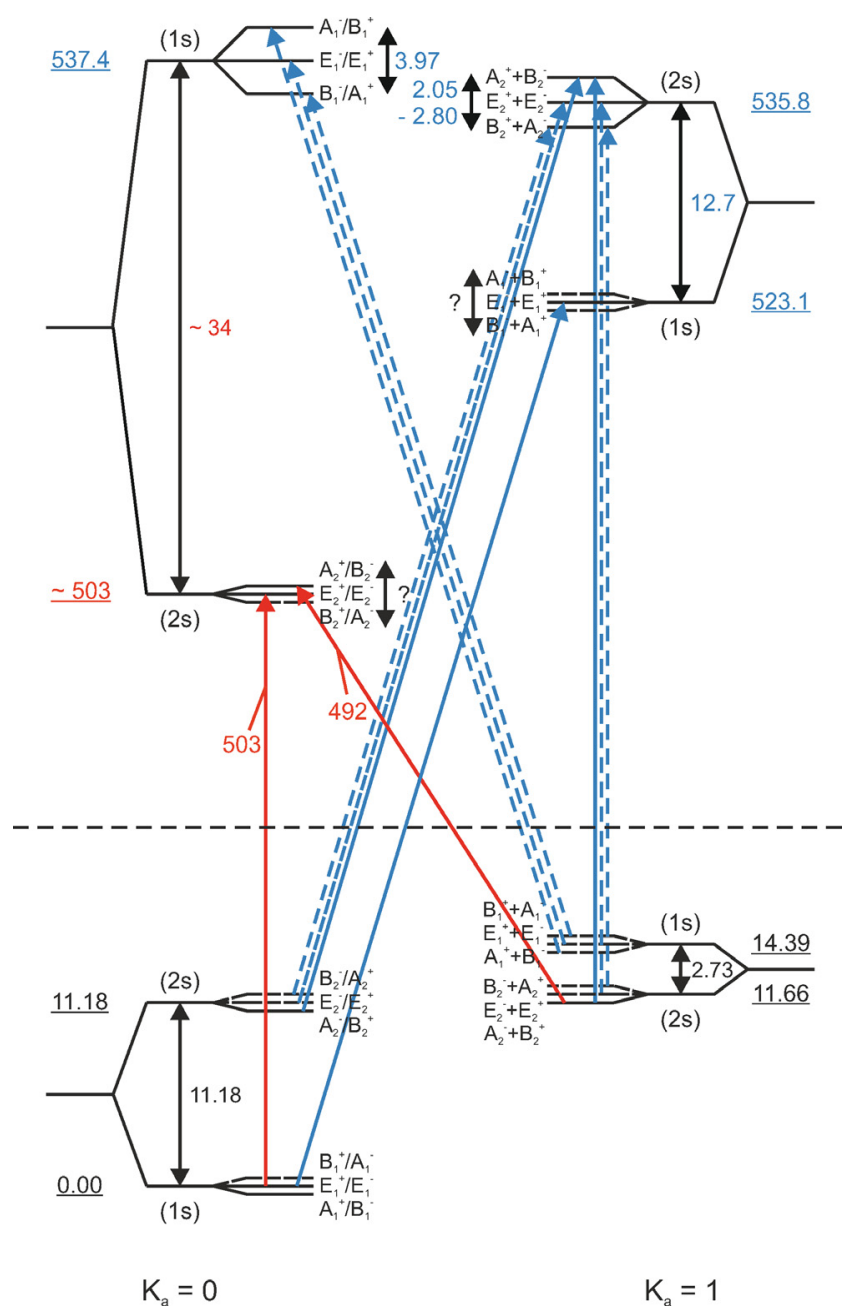

Figure 5. Out-of-plane libration (OPL) VRT energy level diagram. Parallel and perpendicular transitions are shown as vertical and diagonal arrows, respectively; red arrows: observed in the present study; blue arrows: previously observed in gas-phase studies. For bands observed in the present study, the transition frequencies are given (in red). All frequencies and energies are given in $\mathrm{cm}^{-1}$. 
difference between the (1s) and (2s) $K_{\mathrm{a}}=0$ state as $34 \mathrm{~cm}^{-1}$. This corresponds to an increase by a factor of three compared to the ground state, indicating a considerable coupling between the AS tunneling and this intermolecular mode.

\section{Conclusion}

In the present study, a comprehensive spectrum in the low-frequency range is reported, which probes the intermolecular modes of the water dimer. Upon comparison to the previous gas phase studies, we can conclude that the matrix shift of the helium nanodroplet can be neglected. The three tunneling motions are still feasible. We are able to provide for the first time information about the energy of the following intermolecular modes: (2s) $K_{\mathrm{a}}=0$ donor torsion overtone; (2s) $K_{\mathrm{a}}=0$ as well as (1s) $K_{\mathrm{a}}=1$ state of the in-planelibrational mode; as well as (1s) and (2s) states for $K_{\mathrm{a}}=0$ and $K_{\mathrm{a}}=1$ of the out-of-plane librational mode (Table 2). We find that upon excitation of the librational modes, the tunneling splitting is increased considerably compared to the ground state.

This spectrum provides a sensitive test for water dimer potential energy surfaces as well as their dipole moment surfaces. As can be seen in Figure 1, the spectrum predicted from the ab initio $6 d+6 d$ quantum calculation using the HBB2 dimer potential and dipole moment surface is in excellent agreement with our experimental spectrum. The two strong signals observed at 86 and $107-120 \mathrm{~cm}^{-1}$ are attributed to the two bands predicted at 89 and $109 \mathrm{~cm}^{-1}$. The broad signal at $170-195 \mathrm{~cm}^{-1}$ is close to the band predicted at $181 \mathrm{~cm}^{-1}$. Additional bands are predicted at 152 and $209 \mathrm{~cm}^{-1}$, which cannot be assigned unambiguously experimentally, since, this frequency range is dominated by signals attributed to larger water clusters. Bands observed in the frequency range from 265 to $310 \mathrm{~cm}^{-1}$ and from 480 to $510 \mathrm{~cm}^{-1}$ are assigned to the in-plane librational (IPL) and out-of-plane librational (OPL) modes, respectively, which are observed for the first time experimentally.

While for the higher frequency intermolecular modes (IPL and OPL) the predictions from VPT2 calculations ${ }^{[46]}$ are also in good agreement with the experimental band positions, we find a less good agreement for the lower frequency modes, that is, the donor torsion (DT), acceptor wag (AW), acceptor torsion (AT), and intermolecular stretch (OO; see Figure $1 \mathrm{~b}$, lower trace). This can be attributed to the fact, that VPT2 calculations cannot account for the large anharmonicity caused by energy transfer between the intermolecular modes as well as coupling to large-amplitude motions.

We want to note that excellent agreement is found for the spectrum predicted from the quantum calculations using the HBB2 potential and dipole moment surfaces and the experimental spectrum. The excellent agreement is an indication of the high accuracy of the PES and also the dipole moment surface. The predictions based upon the approximate QCMD approach, yield a spectrum that is partly blue-shifted compared to the spectrum using the full $6 \mathrm{~d}+6 \mathrm{~d}$ approach. Furthermore, we want to note, that the intensity of the band at about $300 \mathrm{~cm}^{-1}$ is too low in the QCMD approach. These are likely and (not surprising) an indication that this MD approach is not able to accurately capture the anharmonicity of the potential. We propose that the QCMD approach is too stiff. Especially for the large-amplitude modes, the description lacks the accuracy to reproduce the experimentally observed spectrum.

\section{Acknowledgements}

The experimental work has been supported by the cluster of excellence RESOLV funded by the Deutsche Forschungsgemeinschaft (DFG, German Research Foundation) under

Table 2: Overview of the energy levels of the VRT states of water dimer.

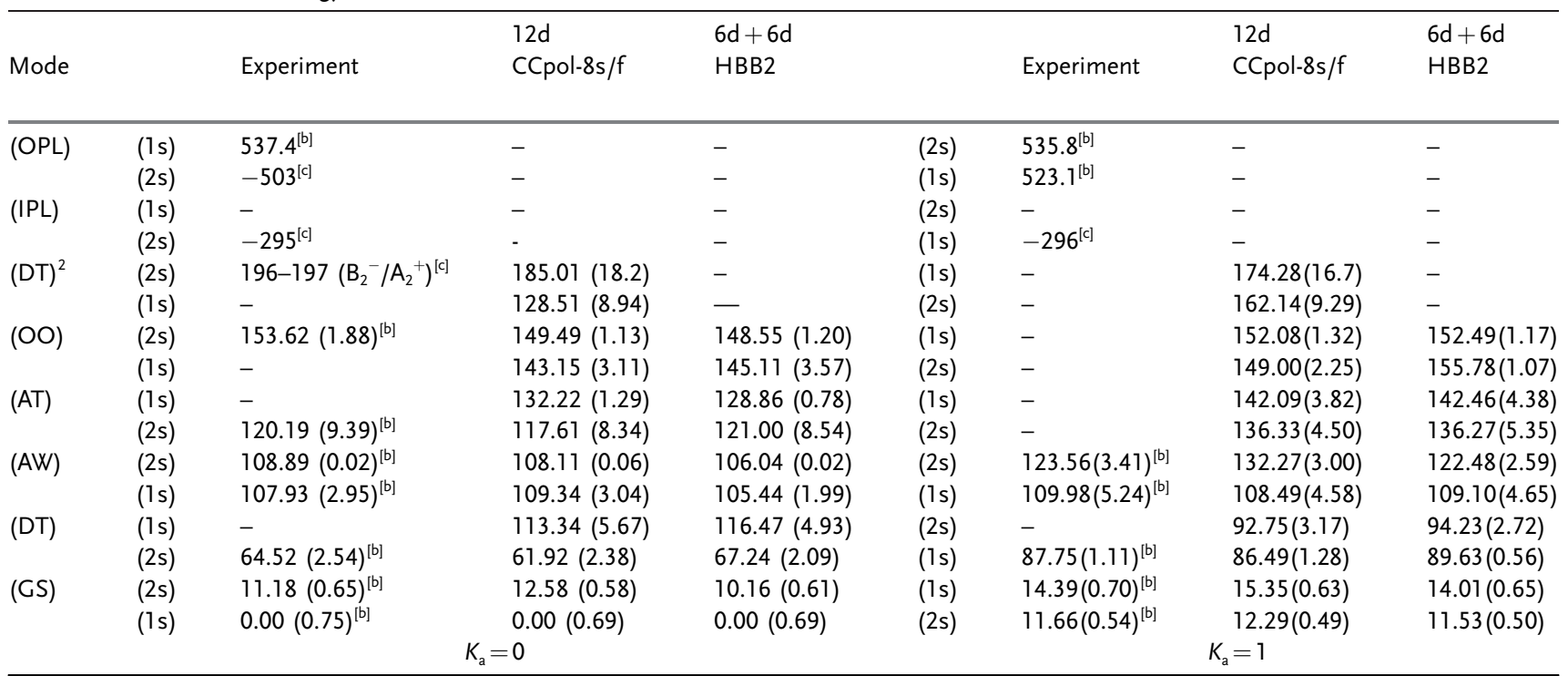

[a] Experimental energies are compared to theoretical predictions ${ }^{[27,50]}$ of the low-frequency VRT levels and tunneling splittings for $\left(\mathrm{H}_{2} \mathrm{O}\right)_{2}$. Values in parentheses denote the size of the interchange tunneling splitting. All values are given in $\mathrm{cm}^{-1}$. [b] Experimental results from previous gas phase studies. ${ }^{[2,31,42-45]}[c]$ From this work. 
Germany's Excellence Strategy -285 EXC-2033-Projektnummer 390677874. The theoretical work at Emory was supported by NASA, Grant No. NNX16AF09G. This work is part of the research programme of the Foundation for Fundamental Research on Matter (FOM), which is part of the Netherlands Organisation for Scientific Research (NWO) and supported by Laserlab-Europe (grant agreement no. 654148, EU-H2020).

\section{Conflict of interest}

The authors declare no conflict of interest.

Keywords: helium nanodroplets - IR spectroscopy . potential surfaces $\cdot$ quantum calculations $\cdot$ water clusters

How to cite: Angew. Chem. Int. Ed. 2019, 58, 13119-13126 Angew. Chem. 2019, 131, 13253-13260

[1] S. S. Xantheas, J. Chem. Phys. 1994, 100, $7523-7534$.

[2] J. K. Gregory, D. C. Clary, J. Phys. Chem. 1996, 100, $18014-$ 18022.

[3] M. P. Hodges, A. J. Stone, S. S. Xantheas, J. Phys. Chem. A 1997, $101,9163-9168$.

[4] G. S. Fanourgakis, S. S. Xantheas, J. Chem. Phys. 2008, 128, 074506.

[5] K. Kitaura, K. Morokuma, Int. J. Quantum Chem. 1976, 10, 325 340.

[6] A. E. Reed, F. Weinhold, J. Chem. Phys. 1983, 78, 4066-4073.

[7] E. A. Cobar, P. R. Horn, R. G. Bergman, M. Head-Gordon, Phys. Chem. Chem. Phys. 2012, 14, 15328-15339.

[8] Y. Mao, P. R. Horn, M. Head-Gordon, Phys. Chem. Chem. Phys. 2017, 19, 5944-5958.

[9] A. Mukhopadhyay, S. S. Xantheas, R. J. Saykally, Chem. Phys. Lett. 2018, 700, 163-175.

[10] C. Millot, A. J. Stone, Mol. Phys. 1992, 77, 439-462.

[11] R. S. Fellers, C. Leforestier, L. Braly, M. Brown, R. Saykally, Science 1999, 284, $945-948$.

[12] E. M. Mas, R. Bukowski, K. Szalewicz, G. C. Groenenboom, P. E. Wormer, A. van der Avoird, J. Chem. Phys. 2000, 113, $6687-6701$.

[13] G. Groenenboom, P. Wormer, A. Van Der Avoird, E. Mas, R. Bukowski, K. Szalewicz, J. Chem. Phys. 2000, 113, 6702-6715.

[14] N. Goldman, R. Fellers, M. Brown, L. Braly, C. Keoshian, C. Leforestier, R. Saykally, J. Chem. Phys. 2002, 116, 10148-10163.

[15] N. Goldman, C. Leforestier, R. Saykally, Philos. Trans. R. Soc. A 2005, 363, $493-508$

[16] C. Leforestier, F. Gatti, R. S. Fellers, R. J. Saykally, J. Chem. Phys. 2002, 117, 8710-8722.

[17] G. S. Tschumper, M. L. Leininger, B. C. Ho man, E. F. Valeev, H. F. Schaefer III, M. Quack, J. Chem. Phys. 2002, 116, 690-701.

[18] R. Bukowski, K. Szalewicz, G. C. Groenenboom, A. Van der Avoird, Science 2007, 315, 1249-1252.

[19] X. Huang, B. J. Braams, J. M. Bowman, J. Phys. Chem. A 2006, $110,445-451$

[20] X. Huang, B. J. Braams, J. M. Bowman, R. E. Kelly, J. Tennyson, G. C. Groenenboom, A. van der Avoird, J. Chem. Phys. 2008, 128,034312 .
[21] A. Shank, Y. Wang, A. Kaledin, B. J. Braams, J. M. Bowman, J. Chem. Phys. 2009, 130, 144314.

[22] B. E. Rocher-Casterline, L. C. Ch'ng, A. K. Mollner, H. Reisler, J. Chem. Phys. 2011, 134, 211101.

[23] Y. Wang, X. Huang, B. C. Shepler, B. J. Braams, J. M. Bowman, J. Chem. Phys. 2011, 134, 094509.

[24] C. Leforestier, K. Szalewicz, A. Van Der Avoird, J. Chem. Phys. 2012, 137, 014305.

[25] W. Cencek, K. Szalewicz, C. Leforestier, R. Van Harrevelt, A. van der Avoird, Phys. Chem. Chem. Phys. 2008, 10, 4716-4731.

[26] V. Babin, C. Leforestier, F. Paesani, J. Chem. Theory Comput. 2013, $9,5395-5403$.

[27] C. Leforestier, Philos. Trans. R. Soc. A 2012, 370, 2675-2690.

[28] G. Garberoglio, P. Jankowski, K. Szalewicz, A. H. Harvey, Faraday Discuss. 2018, 212, 467-497.

[29] F. N. Keutsch, N. Goldman, H. A. Harker, C. Leforestier, R. J. Saykally, Mol. Phys. 2003, 101, 3477-3492.

[30] F. Keutsch, R. Saykally, Proc. Natl. Acad. Sci. USA 2001, 98, $10533-10540$

[31] F. N. Keutsch, L. B. Braly, M. G. Brown, H. A. Harker, P. B. Petersen, C. Leforestier, R. J. Saykally, J. Chem. Phys. 2003, 119, $8927-8937$.

[32] A. Mukhopadhyay, W. T. Cole, R. J. Saykally, Chem. Phys. Lett. $\mathbf{2 0 1 5}, 633,13-26$

[33] D. Laage, J. T. Hynes, Science 2006, 311, 832-835.

[34] J. P. Toennies, A. F. Vilesov, Annu. Rev. Phys. Chem. 1998, 49, 1 41.

[35] C. Callegari, K. K. Lehmann, R. Schmied, G. Scoles, J. Chem. Phys. 2001, 115, 10090-10110.

[36] J. P. Toennies, A. F. Vilesov, Angew. Chem. Int. Ed. 2004, 43, 2622-2648; Angew. Chem. 2004, 116, 2674-2702.

[37] F. Stienkemeier, K. K. Lehmann, J. Phys. B 2006, 39, R127.

[38] M. Choi, G. Douberly, T. Falconer, W. Lewis, C. Lindsay, J. Merritt, P. Stiles, R. Miller, Int. Rev. Phys. Chem. 2006, 25, $15-$ 75.

[39] M. Hartmann, R. Miller, J. Toennies, A. Vilesov, Phys. Rev. Lett. 1995, 75, 1566.

[40] D. Mani, T. Fischer, R. Schwan, A. Dey, B. Redlich, A. Van der Meer, G. Schwaab, M. Havenith, RSC Adv. 2017, 7, $54318-54325$.

[41] R. Schwan, M. Kaufmann, D. Leicht, G. Schwaab, M. Havenith, Phys. Chem. Chem. Phys. 2016, 18, 24063-24069.

[42] E. Zwart, J. Ter Meulen, W. L. Meerts, L. Coudert, J. Mol. Spectrosc. 1991, 147, 27-39.

[43] L. Braly, K. Liu, M. Brown, F. Keutsch, R. Fellers, R. Saykally, J. Chem. Phys. 2000, 112, 10314-10326.

[44] H. Harker, F. Keutsch, C. Leforestier, Y. Scribano, J.-X. Han, R. Saykally, Mol. Phys. 2007, 105, 513-527.

[45] W. T. Cole, R. S. Fellers, M. R. Viant, C. Leforestier, R. J. Saykally, J. Chem. Phys. 2015, 143, 154306.

[46] H. G. Kjaergaard, A. L. Garden, G. M. Chaban, R. B. Gerber, D. A. Matthews, J. F. Stanton, J. Phys. Chem. A 2008, 112, $4324-$ 4335.

[47] T. R. Dyke, J. Chem. Phys. 1977, 66, 492-497.

[48] L. Coudert, J. Hougen, J. Mol. Spectrosc. 1988, 130, 86-119.

[49] L. Coudert, J. Hougen, J. Mol. Spectrosc. 1990, 139, 259-277.

[50] X.-G. Wang, T. Carrington, Jr, J. Chem. Phys. 2018, 148, 074108.

Manuscript received: May 27, 2019

Revised manuscript received: July 23, 2019

Accepted manuscript online: July 27, 2019

Version of record online: August 15, 2019 